\title{
Negative mechanistic reasoning in medical intervention assessment
}

\author{
Jesper Jerkert
}

Division of Philosophy, KTH Royal Institute of Technology, Stockholm, Sweden

This is the author's final version. The article has been published in Theoretical Medicine and Bioethics 36(6), 2015, 425-437. The final publication is available at Springer via http://dx.doi.org/10.1007/s11017-015-9348-2

\begin{abstract}
Traditionally, mechanistic reasoning has been assigned a negligible role in standard EBM (evidence-based medicine) literature, although some recent authors have argued for an upgrade. Even so, the mechanistic reasoning that has received attention has almost exclusively been positive - both in an epistemic sense of claiming that there is a mechanistic chain and in a health-related sense of there being claimed benefits for the patient. Negative mechanistic reasoning has been neglected, both in the epistemic and in the health-related sense. I distinguish three main types of negative mechanistic reasoning and subsume them under a new definition of mechanistic reasoning in the context of assessing medical interventions. This definition is wider than a previous suggestion in the literature. Each negative type corresponds to a range of evidential strengths, and it is argued that there are differences with respect to typical evidential strengths. The variety of negative mechanistic reasoning should be acknowledged in EBM, and it presents a serious challenge to proponents of so-called medical hierarchies of evidence.
\end{abstract}

Keywords: mechanistic reasoning, evidence-based medicine, hierarchy of evidence 


\section{Introduction}

For a couple of decades, mechanistic reasoning has been assigned an insignificant role in clinical medical research. This downgrading coincides with the skeptical attitude towards mechanistic reasoning shown by proponents of evidence-based medicine (EBM). Their attitude can be readily seen in so-called hierarchies of evidence that are put forward in the EBM literature. A hierarchy of evidence is an ordered list of study types (research designs). For a specific research question, the study types at the top of the list are claimed to provide stronger evidence than those further down. For example, hierarchies of evidence have been presented by the OCEBM Levels of Evidence Working Group [1], by Sharon E. Straus et al. [2, p. 169], and by Gordon Guyatt and Drummond Rennie [3, p. 7]. In these, mechanistic reasoning either resides at the bottom of the hierarchy or is absent altogether.

In the last few years, there has been a newly awakened interest in mechanisms and mechanistic reasoning in connection to EBM. EBM supporters, such as Jeremy Howick [4, ch. 10] and Howick, Paul Glasziou, and Jeffrey K. Aronson [5] suggest that mechanistic reasoning should be upgraded. Adam La Caze [6] argues that "basic science," to which mechanistic reasoning certainly belongs, actually plays a more prominent role in EBM than is usually acknowledged by the EBM proponents themselves. Howick, Glasziou, and Aronson [7] discuss the role of mechanisms for extrapolating from study to target populations. Brendan Clarke et al. make convincing arguments for treating evidence of mechanisms in EBM alongside evidence of correlation $[8,9]$. Steven N. Goodman and Jason Gerson present a conceptual framework for evaluating mechanistic evidence in an EBM setting [10]. Holly Andersen argues that mechanistic reasoning, in spite of its general unreliability, has an important role in EBM, viz. in guiding clinicians to apply results from RCTs to individual patients [11]. Robyn Bluhm proposes that the most prominent role of knowledge of mechanisms in EBM is for guiding the design of epidemiological studies, in which the variability is greater than in typical RCTs, where enrolled patients normally do not suffer from multiple diseases or take multiple medications [12]. Barbara Osimani discusses the justification for using mechanistic reasoning and other "low level" evidence in assessments of unintended rather than intended outcomes [13].

Although there is disagreement among these contributions in some respects, they share a common message: mechanistic reasoning has been unfairly underrated in 
EBM, and it is now time to upgrade it according to its real importance. I support this view, and intend to contribute by discussing negative mechanistic reasoning in particular. Mechanistic reasoning can be negative in two different respects: in an epistemic sense and in a health-related sense. Both types of mechanistic reasoning have been largely neglected so far.

In the next section, I discuss how to define mechanistic reasoning. In doing this, I present a number of example situations (scenarios) in which one could discuss whether mechanistic reasoning is being used or not. I also refer to these scenarios later in the text. In the third section, two senses of negative mechanistic reasoning are distinguished, viz. negative in a health-related sense and negative in an epistemic sense. A typology of negative mechanistic reasoning is presented, and the different evidential ranges associated with each type are discussed. In the subsequent section, it is argued that the concept of evidence is taken too narrowly within EBM. Making it wider will have some consequences for so-called hierarchies of evidence. In the final section, the argument is briefly summarized.

\section{Defining mechanistic reasoning}

It is not obvious that "mechanistic reasoning" (or "mechanism-based reasoning") should be the central term of this investigation. Some writers have instead used “evidence of mechanisms" (e.g., [9]) or "knowledge of mechanisms" (e.g., [12]). I

prefer to talk about reasoning because it includes in a more natural way the negative variants that will be the focus in this article. Also, I will not deal with the exact definition of "mechanism," which would perhaps seem more urgent if one were to speak of "evidence of mechanisms" or "knowledge of mechanisms." A recently proposed definition of mechanism by Phyllis McKay Illari and Jon Williamson [14, p. 120] seems to have gained some acceptance, but here, I will leave the issue of an exact definition aside.

To my knowledge, only one definition of mechanistic reasoning in the context of medical intervention assessments has been suggested in the literature so far, namely, by Howick, Glasziou, and Aronson (henceforth, HGA). I will take it as my point of departure:

Mechanistic reasoning is the inference from mechanisms to claims that an intervention produced a patient-relevant outcome. Such reasoning will 
involve an inferential chain linking the intervention (such as antiarrhythmic drugs) with the outcome (such as mortality). [5, p. 434]

I have some objections to this HGA definition and will argue for a wider definition. In doing that, my main attitude will be that a wider definition gives a more interesting class of phenomena, all of which are similar enough to share a common label, here conveniently taken to be "mechanistic reasoning". At some points, however, I will instead adopt the attitude that the meanings of "mechanistic" and "reasoning" must be allowed to constrain what should count as mechanistic reasoning.

The HGA definition speaks of a "patient-relevant" outcome. There is no elaboration on what this means in [5], but Howick, reproducing an almost identical definition of mechanistic reasoning, defines a patient-relevant outcome as "one that makes people feel better or live longer" [4, p. 128]. In his view, only outcomes that are good to the patient are patient-relevant. Obviously, this is narrower than outcomes that could matter to the patient.

I find Howick's restriction counter-intuitive. An inference that a certain treatment will probably make patients feel worse or live shorter seems patientrelevant, too. Perhaps, then, a patient-relevant outcome should rather be defined as "one that makes people feel better/worse or live longer/shorter." However, this is still not exactly right. It would mean that a no-effect outcome is not patient-relevant. That is questionable. An inference that a particular treatment probably has no effect on a particular disease or collection of symptoms seems highly patient-relevant.

My conclusion is that patient-relevance should not, in this context, be related to effect size or to the question of whether the patient gets better or worse (or neither). I would exchange "a patient-relevant outcome" for "a specified intervention outcome." I am trying to explicate what is meant by mechanistic reasoning in a special context, that of medical intervention assessments. Therefore, the discussion should be restricted to intervention outcomes, i.e., outcomes that are (believed to be) caused by the intervention. ${ }^{1}$ An outcome has to be specified; otherwise, inferring that an intervention will have "some" outcome without being able to tell what it is could count as mechanistic reasoning. Such imprecise reasoning should not be included.

\footnotetext{
${ }^{1}$ Of course, an inference about an outcome not related to a medical intervention, e.g., an inference referring to the laws of gravitation, could be due to mechanistic reasoning, but hardly in the present context of interest.
} 
Let me introduce a few simple scenarios to illustrate my points so far and to inform later discussions about the meaning of mechanistic reasoning. Here is the first scenario, Scenario PR:

Drug $A$ leads to an increase of measurable and wanted outcome $C$ via a mechanistic chain and simultaneously, through another mechanistic chain, to a decrease of $C$ of approximately equal size. In sum, therefore, it is believed that there is approximately no change of $C$. The two mechanistic chains overlap partly.

Clarke et al. have discussed something very similar and called it "the masking problem" [8]. The scenario is no mere theoretical construction. Daniel Steel notes that increased exercise results in more calories being burned, which should lead to weight loss $[15$, p. 68]. On the other hand, exercise also stimulates one's appetite, which should lead to larger amounts of food intake and hence to weight gain. In principle, one could imagine these two effects cancelling each other out. (In reality, it is clear that the weight loss effect is believed to be the larger one.) Another example could be Germund Hesslow's account that oral contraceptives could cause an increased probability of thrombosis but simultaneously decrease the probability of pregnancy, and pregnancy is known to increase the probability of thrombosis [16]. Hence, through different mechanistic chains, but partly overlapping, since they have the same starting point and the same outcome variable, oral contraceptives both increase and decrease the probability of thrombosis. I claim that Scenario PR exemplifies mechanistic reasoning, but this could be denied by someone requiring patientrelevance in terms of a substantial effect.

Here is another scenario, Scenario REV:

Injection into the blood of substance $A$ is argued to lead mechanistically to strengthening of one of the symptoms associated with medical condition $C$. For the other symptoms associated with $C$, mechanistic reasoning does not suggest any effect. But what we really want, for the benefit of the patient, is a decrease of the symptoms associated with $C$. 
The fact that reasoning involving mechanisms leads to claims of an outcome that is the reversal of what is desired should not stop us from calling the reasoning mechanistic, but that would be the consequence of adopting Howick's notion of patient-relevance.

I believe another change must be made in the HGA definition. It speaks of "claims that an intervention produced a patient-relevant outcome" (emphasis added). The production requirement is too strong. For consider the following scenario, Scenario FAIL:

A new drug with purportedly active agent $A$ is being marketed as relieving a specific condition $C$. The drug is to be ingested orally. Much is known about $A$, both in terms of what happens to $A$ chemically in the human body and in terms of other observable effects on the body. (But there is no complete knowledge of what happens to $A$ when ingested orally, because-if for no other reason-we still do not know all details about its metabolism in humans.) Quite a lot is known about $C$, too. We can describe $C$ very accurately, and for each of the features that constitute $C$, we believe that we know what immediately brings it about. (However, we do not know everything there is to know about how these states that bring about the constituents of $C$ are themselves brought about.) From our rich but incomplete knowledge about $A$ and $C$, we try to find a credible — or at least possible - way of understanding or charting how a relief of $C$ can be brought about by oral intake of $A$. But in spite of serious attempts to find a causal chain from $A$ to not- $C$, we fail to find one.

According to the HGA definition, there is no mechanistic reasoning in Scenario FAIL, since no inference from mechanisms was made and since, in the end, no claim was made that the intervention produced an outcome. But I think Scenario FAIL should count as mechanistic reasoning. What intuitively seems important for reasoning to be called mechanistic is that one tries seriously to find mechanisms, not that one actually succeeds. An analogous situation could be to perform experimental work. Seriously 
trying to perform a scientific experiment would, to most of us, count as experimental work even though the experiment would turn out to be a methodological failure. ${ }^{2}$

For an attempt to find a mechanistic chain to count as mechanistic reasoning, however, there should be a non-negligible chance of success. (This is what is meant by saying that one should try seriously to find mechanisms.) It does not seem right to say that one is performing mechanistic reasoning that attempts to connect an intervention and an outcome if the effort is completely pointless from the point of view of previously acquired knowledge. For example, if I am told that a pill branded $\mathrm{X}$ is good at relieving headache, it will be futile to try to come up with a mechanistic account of why $\mathrm{X}$ is helpful if I am completely ignorant of what X contains.

Intervention and outcome must both be somehow connected to a web of previous knowledge for mechanistic reasoning to be meaningful, for it is only if both intervention and outcome are part of such a web of knowledge that they can possibly be connected mechanistically. I believe that my point here is akin to that of Clarke and co-authors, who prefer the term "evidence of mechanisms," when they insist that there must be evidence and not just "stories" [9, p. 350].

Now consider the following scenario, Scenario LED:

\begin{abstract}
A headset with light-emitting diodes is to be used for "channeling bright light directly to photosensitive regions of the brain through the ear canal" for a few minutes per day. This is claimed to be an efficient treatment of mood swings due to, e.g., seasonal daylight variations. ${ }^{3}$ However, according to current physiological knowledge, there are no photoreceptors in the ear canal, and hence, any light entered there can only be mediated as heat to other parts of the head. There is no conceivable way in which small amounts of heat in the ear canal could causally decrease the frequency or severity of mood swings. In conclusion, there cannot be a mechanistic connection between intervention and postulated outcome in this case.
\end{abstract}

\footnotetext{
${ }^{2}$ It could be noted that several writers seem to take for granted that certain reasoning in which no mechanism is presented still qualifies as mechanistic. For example, Howick, Glasziou, and Aronson in spite of the denial in their own definition that such reasoning is mechanistic, write that "[m]any EBM proponents accept mechanistic reasoning ... for ... ruling out implausible hypotheses" [5, p. 433]. ${ }^{3}$ Quotation and medical claims taken from a manufacturer's webpage, www2.valkee.com/uk/ [accessed 18 September, 2014].
} 
In Scenario LED, no real attempt is made to find a mechanistic chain. Instead, "metamechanistic reasoning" is performed, indicating that there cannot be a mechanism that connects the intervention with the outcome. Contra the HGA definition, in Scenario LED, there is no "inference from mechanisms," nor any claim that the intervention produced an outcome. But again, it seems legitimate to count this as mechanistic reasoning. Although the reasoning is performed at a meta-level, the goal is identical to that of "ordinary" mechanistic reasoning, namely, to assess the causal link between intervention and clinical outcome. And there is a serious attempt to find a link, since the reasoning involves investigating its possibility according to our current knowledge. Therefore, the reasoning in Scenario LED should count as mechanistic reasoning.

By meta-mechanistic reasoning, I mean, more precisely, reasoning in which the question of interest is whether there could be a mechanistic chain from an intervention to specified outcomes or not. A positive answer is not in itself very interesting; one would then go on to discuss exactly how the chain is constituted. Admittedly, the boundary between meta-mechanistic reasoning and "ordinary" mechanistic reasoning is rather unclear in that case. A negative answer, by contrast, could be very informative, as exemplified by Scenario LED.

Meta-mechanistic reasoning may involve appeals to theories as well as to empirical findings. In Scenario LED, both types of appeal are found: appeal to the empirical findings that there are no photoreceptors in the ear canal, to theories about plausible energy conversions from light to other forms, and to theories about the possible effects of small amounts of heat on human tissue.

There is one particular type of reasoning that investigates whether there could be a mechanistic chain from intervention to specified outcomes but should nevertheless not count as mechanistic reasoning. Consider the following scenario, Scenario BACK:

Proponents of a certain intervention $I$ claim that it leads to beneficial outcomes $O$, with an as of yet unknown mechanistic chain. Many empirical studies of the effect of $I$ on $O$ have been reported, however, and their overall message is that $I$ does not affect $O$. A critic concludes that there is probably no mechanistic chain from $I$ to $O$. 
The critic is right, of course: there is probably no mechanistic chain from $I$ to $O$. If the empirical results were positive, one would have concluded that there is probably a mechanistic chain from $I$ to $O$. But in neither case should this count as mechanistic reasoning. This is because the only argument presented for or against the claim that there is a mechanistic chain from $I$ to $O$ is an appeal to purely empirical investigations of the very same chain from $I$ to $O$ (i.e., the effect of $I$ on $O$ ). Mechanistic reasoning is normally considered to be distinct from empirical investigations, but this distinction becomes too blurred if one allows empirical results relating $I$ and $O$ to pose, by themselves, as mechanistic reasoning from $I$ to $O$. On the other hand, empirical investigations of the effect of $I$ on some intermediate step in the chain from $I$ to $O$ could be part of genuine mechanistic reasoning.

Scenario BACK also illustrates the simple fact that when much is known empirically about the effects of $I$ on $O$, there is rarely a need for mechanistic reasoning about the plausibility that $I$ has an effect on $O$. (But mechanistic reasoning can be useful for assessing the effect of $I$ on other outcomes, such as unwanted side effects.)

From the above considerations, I will now propose a new definition of mechanistic reasoning in the context of medical intervention assessments, deviating from the HGA definition. The proposal runs as follows:

Mechanistic reasoning is reasoning that involves either an inference from mechanistic chains to claims concerning specified intervention outcomes, or an inference from an investigation of whether there are plausible mechanistic chains to claims concerning specified intervention outcomes.

In this definition, neither the plural "from mechanisms" nor the plural "specified intervention outcomes" should be given too literal interpretations. I do not intend to say that reasoning that includes only one mechanism or only one specified outcome cannot be mechanistic. Also, an "investigation of whether there are plausible mechanistic chains" cannot consist solely in empirical studies of the effect of the intervention on the specified outcomes. 


\section{A typology of negative mechanistic reasoning}

I now turn to the question of what could be meant by positive and negative mechanistic reasoning, and how to characterize various types of negative mechanistic reasoning.

In the context of medical intervention assessments, the underlying goal is to prevent diseases, to cure patients from diseases, or to relieve the patients' unwanted symptoms. In a health-related sense, then, positive mechanistic reasoning is mechanistic reasoning that points towards the fulfillment of this goal, whereas in negative mechanistic reasoning, the outcome would be bad to the patient. There is a middle option, too: the outcome may be neutral, either in the form of several conflicting outcomes that cancel out or in the form of no believed outcome at all. For the purpose of the present article, I count mechanistic reasoning with a neutral outcome as negative if performed against background beliefs of beneficial effects, and as positive if performed against background beliefs of harmful effects.

There is also another sense in which one could talk about positive and negative mechanistic reasoning, and that is epistemically:

Mechanistic reasoning is positive in an epistemic sense if, and only if, it claims that there is a mechanistic chain.

And its opposite:

Mechanistic reasoning is negative in an epistemic sense if, and only if, it claims that there is no mechanistic chain.

There is no middle ground here; mechanistic reasoning must contain a claim about either the existence or the absence of a mechanistic chain. Of the mechanistic reasoning scenarios presented so far, PR and REV are epistemically positive, whereas FAIL and LED are negative.

The epistemic and health-related senses of positive and negative mechanistic reasoning are totally different, but both are relevant for understanding the variety of mechanistic reasoning and for understanding how this variety has been largely neglected. For in fact, mechanistic reasoning that is positive in both senses has been very predominant in the discussions, both for showing that mechanistic reasoning can 
be reliable under certain circumstances and for exemplifying that it may be unreliable. Negative mechanistic reasoning, in either or both of the senses defined, has only been mentioned in passing.

I now introduce the label "NegA" for mechanistic reasoning that is negative in the health-related sense, but positive in the epistemic sense. In other words, NegA reasoning includes a mechanistic chain but suggests a negative (or at least a nonpositive) outcome to the patient. Then, NegA reasoning is in many respects a mirror image of positive mechanistic reasoning. Positive mechanistic reasoning (i.e., reasoning that is positive in both senses) is known to be associated with a very varying degree of evidential strength, as demonstrated by the fact that discussants have been able to point to examples of such reasoning to claim alternately that it can be, under certain circumstances, very reliable or that it can be, under other circumstances, very unreliable. NegA reasoning inherits this variation in evidential strength. When we know (or think that we know) a lot about the mechanistic chain under consideration, either from empirical studies or from more theoretical deliberations, we ascribe NegA reasoning great evidential strength; when our knowledge is scarce and uncertain, NegA reasoning becomes evidentially weak.

\begin{tabular}{|l|l|l|l|}
\hline Label & Characterization & $\begin{array}{l}\text { Evidential } \\
\text { strength }\end{array}$ & Example \\
\hline NegA & $\begin{array}{l}\text { Reasoning which includes a } \\
\text { mechanistic chain, suggesting a } \\
\text { negative outcome to the patient } \\
\text { (or a neutral outcome against a } \\
\text { background expectation of a } \\
\text { positive outcome) }\end{array}$ & $\begin{array}{l}\text { Low, } \\
\text { intermediate, or } \\
\text { high }\end{array}$ & Scenario REV \\
\hline NegB & $\begin{array}{l}\text { Reasoning that constitutes a } \\
\text { serious but failed attempt to find a } \\
\text { mechanistic chain connecting the } \\
\text { intervention and the outcome. }\end{array}$ & $\begin{array}{l}\text { Low or } \\
\text { intermediate }\end{array}$ & $\begin{array}{l}\text { Scenario } \\
\text { FAIL }\end{array}$ \\
\hline NegC & $\begin{array}{l}\text { Reasoning in which meta- } \\
\text { mechanistic arguments suggest } \\
\text { that there cannot be a mechanistic } \\
\text { connection between intervention } \\
\text { and outcome. }\end{array}$ & $\begin{array}{l}\text { Intermediate or } \\
\text { high }\end{array}$ & Scenario LED \\
\hline
\end{tabular}

Table 1. Types of negative mechanistic reasoning.

As for mechanistic reasoning that is negative in the epistemic sense, I discuss two main types, that I call "NegB" and "NegC". In both, the claim is that there is no 
(plausible) mechanistic chain. To distinguish them, one must ask: Are we trying but failing to find a mechanistic chain? Or are we using meta-mechanistic reasoning to argue that there could not be a chain? If the answer is yes to the first question, NegB reasoning is being used; if the answer is yes to the second, $\mathrm{NegC}$ is being used. The benefit of making this distinction is that $\mathrm{NegB}$ and $\mathrm{NegC}$ reasoning will be associated with different evidential strengths. A summary of types NegA, NegB, and NegC is given in Table 1.

Before discussing the difference in evidential strength between NegB and $\mathrm{NegC}$ reasoning, two remarks are appropriate. First, it is the typical strength of evidence that is of interest here, but of course, there is room for some variation. Secondly, it needs to be emphasized that the strength of evidence indicated here is the one pertaining to the outcome that was specified in the reasoning. This is not necessarily an outcome with which one can assess the overall efficiency of the intervention on an underlying disease. For example, in Scenario PR, there is some "measurable outcome $C$ " but it is unclear whether this is the only outcome of interest for the overall cure of the disease (or the overall relief of symptoms). One therefore has to be cautious if one wants to make an inference from mechanistic reasoning to the possibility of relieving all symptoms associated with the disease. On the other hand, in Scenario LED the outcome is given in such a way that the inference from mechanistic reasoning applies to the overall treatment effect.

Why is the evidential strength of NegB typically low or intermediate? The main reason is that failing to find a mechanistic chain does not at all guarantee that there is none. Even though $\mathrm{NegB}$ reasoning does not correspond to complete ignorance (since, by definition, NegB reasoning involves a serious search for a mechanistic chain), it does correspond to a substantial ignorance in the sense that one does not find oneself in a situation where one can appeal to well-established theories that would rule out the existence of a mechanistic chain. For if one could appeal to such theories, one would be performing $\mathrm{NegC}$ reasoning. Since $\mathrm{NegC}$ is principled and supposedly based on previous knowledge, which in many cases may be considered to have a high degree of certainty, it is reasonable to assign a higher typical strength of evidence to NegC reasoning than to NegB reasoning. However, there is room for variation within $\mathrm{NegC}$ reasoning as well, and therefore, its strength of evidence is marked not as "High" but as "Intermediate or high." 
I would like to stress that the purported absence of a plausible mechanistic chain does not, according to my definition, qualify as mechanistic reasoning unless it can be subsumed under types NegB or NegC. So, for example, when Andrew J. Turner writes that there are several examples of "treatments that are known to be efficacious, but for which there is limited understanding of the mechanistic model behind them," and mentions general anaesthetics as the prime example [17, p. 46f], this constitutes mechanistic reasoning only insofar as either there has been a serious attempt to find a mechanistic chain (NegB) or meta-mechanistic reasoning suggests there cannot be any $(\mathrm{NegC})$. Sometimes it is not clear which type (if any) an "absence of evidence" statement belongs to. A negative mechanistic statement is not by itself negative mechanistic reasoning.

Further distinctions, yielding subtypes to $\mathrm{NegA}, \mathrm{NegB}$, and $\mathrm{NegC}$, would surely be relevant in some situations. For example, $\mathrm{NegC}$ would have been split into several types had one wished to distinguish between different types of metamechanistic arguments that could be invoked. Here, I will not pursue further subdivisions but will be content with NegA, NegB, and NegC as discussed.

Could combinations of NegA, NegB, and $\mathrm{NegC}$ be used correctly for the same intervention/outcome pair? Yes, but only the combination $\mathrm{NegB}+\mathrm{NegC}$, and in that case, we will have to allow for some fallibility in the reasoning. For if $\mathrm{NegC}$ is used and is correct, then, strictly speaking, there is no point in even trying to find a mechanistic chain (which is done in NegB); there cannot be one. But if one allows for some uncertainty as to whether the $\mathrm{NegC}$ reasoning is really correct, then it may be reasonable to combine $\mathrm{NegC}$ and $\mathrm{NegB}$, i.e., both saying that there cannot be a mechanistic chain and trying seriously but failing to find one.

$\mathrm{NegA}$ reasoning cannot be combined with any of $\mathrm{NegB}$ or $\mathrm{NegC}$, because NegA is epistemically positive whereas NegB and NegC are epistemically negative.

\section{The nature of evidence and consequences for hierarchies of evidence}

My presentation of the types NegA, NegB, and NegC is offered as a first step of systematization in the discussion about negative mechanistic reasoning. As for positive mechanistic reasoning and the circumstances under which it is reliable, several important contributions have been made recently, although they do not yet demonstrate a consensus $[4,9,11,12]$. Further analyses are needed for a fuller 
appreciation of the evidential roles that could be played by mechanistic reasoning, both positive and negative. Already by now, however, one can say some important things regarding what should constitute evidence within EBM.

As has been argued above, some types of mechanistic reasoning can provide strong evidence. Mechanistic reasoning in the $\mathrm{NegC}$ format, with appeals to theories that undermine the credibility of the intervention, can be very convincing. Indeed, objections to homeopathy and other implausible medical schools of thought have been put forward along these lines so frequently that it is rather their absence that may cause raised eyebrows.

For example, critique has been directed at the Cochrane Collaboration for ignoring non-RCT evidence, e.g., mechanistic reasoning, when assessing the available evidence concerning certain treatments. In a Cochrane review of homeopathy for dementia, it was concluded that the absence of RCTs prevented any conclusions on its effectiveness. Two critics asked, "Is it really not possible to comment on the use of homeopathy in dementia without an RCT?" [18].

In another example, a Cochrane review [19] failed to take any theoretical and mechanistic considerations into account in the aggregation of available evidence on the effectiveness of acupressure on postoperative nausea and vomiting (PONV). Not even under the heading "Implications for research" did the report authors acknowledge any need for a better understanding of how stimulation of the P6 acupressure point could possibly lead to a relief of PONV, which they claimed to have found. The failure to take any mechanistic considerations into account in such a case makes a strange impression.

The fundamental question here is what should count as evidence in EBM. There seem to be two notions of evidence around. One is very broad: anything that justifies belief is evidence [20]. A narrower notion results from emphasizing that evidence should be empirical. In EBM textbooks, the concept of evidence is definitely taken to be the narrow one. Gordon Guyatt and Drummond Rennie, for example, write, "What is the nature of the 'evidence' in EBM? We suggest a broad definition: any empirical observation about the apparent relation between events constitutes potential evidence" [3, p. 6]. In spite of the authors' assertion that the definition is "broad," it seems to be too narrow to include mechanistic reasoning, judging from its negligible role in their book. The textbook by Straus et al. [2] gives no explicit 
definition of evidence, but from the authors' discussion, it seems that mechanistic reasoning would not qualify. The same is true for Jorgen Nordenstrom's textbook [21].

This attitude has to be changed within EBM, since it obscures the fact that mechanistic reasoning and other theoretical deliberations may constitute important evidence. Upgrading theoretical deliberations will have an impact on the credibility of hierarchies of evidence, however. As was mentioned in the first section above, currently used hierarchies of evidence typically include mechanistic reasoning at the bottom or not at all. These hierarchies of evidence make sense, if interpreted cautiously, ${ }^{4}$ but they seem to do so only if:

(1) action-guidance is considered more important than causality,

(2) direct empirical evidence is considered more important than theoretically framed evidence, and

(3) positive claims are considered more interesting than negative claims.

One could endorse, or at least accept, (1) in medical intervention research, but (2) and (3) are highly questionable. As I have argued, theoretical deliberations may yield strong evidence, and negative claims may be highly patient-relevant.

Several commentators have noted that currently promoted hierarchies of evidence are not suitable for causal inferences. Jacob Stegenga has gone even further by proposing that hierarchies of evidence be abandoned altogether [22]. Others, e.g., Sven Ove Hansson, suggest that they may still be useful for action guidance [23]. To give a verdict on the overall use of hierarchies of evidence in medical intervention research is beyond the scope of the present article. I note, however, that already by distinguishing NegA, NegB, and NegC mechanistic reasoning and claiming that they have different typical evidential strengths, and by arguing that these variants of negative mechanistic reasoning should be recognized as evidence in medical intervention research, I have presented supporters of hierarchies of evidence with a serious challenge. If mechanistic reasoning could have very different evidential strengths, then, presumably, different types of mechanistic reasoning should be entered at different levels in the hierarchy of evidence. Designing and justifying useful hierarchies of evidence would then seem to be a formidable task.

\footnotetext{
${ }^{4}$ For example, they should not be interpreted to entail that a single study of a type higher up in the hierarchy always outweighs any number of studies belonging to types further down.
} 


\section{Conclusion}

Briefly, I summarize my arguments as follows. Mechanistic reasoning has been neglected for too long within EBM. This is particularly true for negative mechanistic reasoning. I have offered a basic categorization of negative mechanistic reasoning into types NegA, NegB, and NegC. NegA is distinguished from NegB and NegC reasoning by including an actual mechanistic chain. $\mathrm{NegB}$ and $\mathrm{NegC}$ reasoning are distinguished with regard to whether there is an attempt to find a mechanistic chain or not. The three negative types are associated with different typical strengths of evidence. Giving mechanistic reasoning its due attention in EBM will require the widening of what constitutes evidence as compared to what can be found in textbooks by EBM proponents. This, in turn, will present difficulties for the design of hierarchies of evidence, if they are to be retained.

\section{References}

1. OCEBM Levels of Evidence Working Group. 2011. The Oxford Centre for Evidence-Based Medicine 2011 levels of evidence, http:/www.cebm.net/wp-content/uploads/2014/06/CEBMLevels-of-Evidence-2.1.pdf. Accessed Nov. 5, 2015.

2. Straus, S.E., W.S. Richardson, P. Glasziou, and R.B. Haynes. 2005. Evidence-based medicine: how to practice and teach EBM. $3^{\text {rd }}$ ed. Edinburgh: Elsevier.

3. Guyatt, G., and D. Rennie (eds.). 2002. Users guides to the medical literature: a manual for evidence-based clinical practice. Chicago: American Medical Association Press.

4. Howick, J. 2011. The philosophy of evidence-based medicine. Wiley-Blackwell \& BMJ Books.

5. Howick, J., P. Glasziou, and J.K. Aronson. 2010. Evidence-based mechanistic reasoning. Journal of the Royal Society of Medicine 103: 443-441.

6. La Caze, A. 2011. The role of basic science in evidence-based medicine. Biology \& Philosophy 26(1): 81-98.

7. Howick, J., P. Glasziou, and J.K. Aronson. 2013. Can understanding mechanisms solve the problem of extrapolating from study to target populations (the problem of 'external validity')? Journal of the Royal Society of Medicine 106: 81-86.

8. Clarke, B., D. Gillies, P. Illari, F. Russo, and J. Williamson. 2013. The evidence that evidencebased medicine omits. Preventive Medicine 57(6): 745-747.

9. Clarke, B., D. Gillies, P. Illari, F. Russo, and J. Williamson. 2014. Mechanisms and the evidence hierarchy. Topoi 33(2): 339-360.

10. Goodman, S.N. and J. Gerson. 2013. Mechanistic evidence in evidence-based medicine: $a$ conceptual framework. Agency for Healthcare Research and Quality. AHRQ Publication No. 13EHC042-EF. http://www.ncbi.nlm.nih.gov/books/NBK154584/pdf/Bookshelf_NBK154584.pdf. Accessed Nov. 5, 2015.

11. Andersen, H. 2012. Mechanisms: what are they evidence for in evidence-based medicine? Journal of Evaluation in Clinical Practice 18(5): 992-999.

12. Bluhm, R. 2013. Physiological mechanisms and epidemiological research. Journal of Evaluation in Clinical Practice 19(3): 422-426.

13. Osimani, B. 2014. Hunting side effects and explaining them: should we reverse evidence hierarchies upside down? Topoi 33(2): 295-312.

14. Illari, P.M., and J. Williamson. 2012. What is a mechanism? Thinking about mechanisms across the sciences. European Journal for Philosophy of Science 2: 119-135.

15. Steel, D. 2008. Across the boundaries: extrapolation in biology and social science. Oxford: 
Oxford University Press.

16. Hesslow, G. 1976. Two notes on the probabilistic approach to causality. Philosophy of Science 43(2): 290-292.

17. Turner, A.J. 2012. Evidence based medicine, 'placebos' and the homeopathy controversy. $\mathrm{PhD}$ Diss., University of Nottingham.

18. Hammerstrøm, K.T., and A. Bjørndal. 2011. If there are no randomised controlled trials, do we always need more research? [editorial]. Cochrane Database of Systematic Reviews. http://www.cochranelibrary.com/editorial/10.1002/14651858.ED000024. Accessed Nov. 5, 2015.

19. Lee, A., and L.T.Y. Fan. 2009. Stimulation of the wrist acupuncture point P6 for preventing postoperative nausea and vomiting. Cochrane Database of Systematic Reviews 2009(2): CD003281.

20. Kelly, T. 2006. Evidence. In The Stanford Encyclopedia of Philosophy (Fall 2008 Edition), ed. E.N. Zalta. http://plato.stanford.edu/archives/fall2008/entries/evidence/. Accessed Nov. 5, 2015.

21. Nordenstrom, J. 2007. Evidence-based medicine in Sherlock Holmes' footsteps. Malden, MA: Blackwell Publishing.

22. Stegenga, J. 2014. Down with the hierarchies. Topoi 33(2): 313-322.

23. Hansson, S.O. 2014. Why and for what are clinical trials the gold standard? Scandinavian Journal of Public Health 42(Suppl. 13): 41-48. 